\title{
COUNTING UNLABELLED SUBTREES OF A TREE IS \#P-COMPLETE
}

\author{
LESLIE ANN GOLDBERG AND MARK JERRUM
}

\begin{abstract}
The problem of counting unlabelled subtrees of a tree (that is, subtrees that are distinct up to isomorphism) is \#P-complete, and hence equivalent in computational difficulty to evaluating the permanent of a 0,1 -matrix.
\end{abstract}

\section{Introduction}

Valiant's complexity class \#P (see [11]) stands in relation to counting problems as NP does to decision problems. A function $f: \Sigma^{*} \rightarrow \mathbb{N}$ is in \#P if there is a nondeterministic polynomial-time Turing machine $M$ such that the number of accepting computations of $M$ on input $x$ is $f(x)$, for all $x \in \Sigma^{*}$. A counting problem, that is, a function $f: \Sigma^{*} \rightarrow \mathbb{N}$, is said to be \#P-hard if every function in \#P is polynomial-time Turing reducible to $f$; it is complete for \#P if, in addition, $f \in$ \#P. A \#P-complete problem is equivalent in computational difficulty to such problems as counting the number of satisfying assignments to a Boolean formula, or evaluating the permanent of a 0,1-matrix, which are widely believed to be intractable. For background information on \#P and its completeness class, refer to one of the standard texts, for example $[3,8]$.

The main result of the paper-advertised in the abstract, and stated more formally below-is interesting on two counts. First, it provides a rare example of a natural question about trees that is unlikely to be polynomial-time solvable. (Two other examples are determining a vertex ordering of minimum bandwidth $[1,4]$, or determining the 'harmonious chromatic number' [2].) Second, it is, as far as we are aware, the first intractability result concerning the counting of unlabelled structures.

Some definitions follow. By rooted tree $(T, r)$ we simply mean a tree $T$ with a distinguished vertex $r$, the root. An embedding of a tree $T^{\prime}$ in a tree $T$ is a injective map $\iota$ from the vertex set of $T^{\prime}$ to the vertex set of $T$ such that $(\iota(u), \iota(v))$ is an edge of $T$ whenever $(u, v)$ is an edge of $T^{\prime}$. Sometimes $T^{\prime}$ and $T$ will be rooted, in which case we may insist that $\iota$ maps the root $r^{\prime}$ of $T^{\prime}$ to the root $r$ of $T$. We now define a sequence of problems leading to one of interest; we do not claim that both the intermediate problems are particularly natural.

Name. \#BIPARTITEMATCHINGS.

Instance. A bipartite graph $G$ with $n$ vertices in each of its two vertex sets.

Output. The number of matchings of all sizes in $G$.

Name. \#COMmonRootedSubTREes.

Instance. Two rooted trees, $\left(T_{1}, r_{1}\right)$ and $\left(T_{2}, r_{2}\right)$.

This work was supported in part by the ESPRIT Working Group 21726 'RAND2' and by EPSRC grant GR/L60982. Received 29 November 1999, revised 20 March 2000; published 26 April 2000. 2000 Mathematics Subject Classification 68Q25, 68R10, 05C05, 05C30, 05C60 (C) 2000, Leslie Ann Goldberg and Mark Jerrum 
Output. The number of distinct (up to isomorphism) rooted trees $(T, r)$ such that $(T, r)$ embeds in $\left(T_{1}, r_{1}\right)$ and $\left(T_{2}, r_{2}\right)$ with $r$ mapped to $r_{1}$ and $r_{2}$, respectively.

Name. \#RootedSubTREes.

Instance. A rooted tree, $(T, r)$.

Output. The number of distinct (up to isomorphism) rooted trees $\left(T^{\prime}, r^{\prime}\right)$ such that $\left(T^{\prime}, r^{\prime}\right)$ embeds in $(T, r)$ with $r^{\prime}$ mapped to $r$.

Name. \#SUBTREES.

Instance. A tree $T$.

Output. The number of distinct (up to isomorphism) subtrees of $T$.

We will use each of the problem names in an obvious way to denote a function from instances to outputs: thus \#RootedSubtrees $(T, r)$ denotes the number of distinct rooted subtrees of the rooted tree $(T, r)$. Our main result is the following.

Theorem 1. \#SubTrees is \#P-complete.

Proof. The \#P-hardness of \#BIPARTITEMatchings follows from Valiant's paper [11]. In particular, Valiant shows that the problem IMPERFECTMATCHINGS is \#P-complete. IMPERFECTMATCHings is the same as \#BIPARTITEMATCHINGS except that the size of the two vertex sets may differ. IMPERFECTMATCHINGS may be reduced to \#BIPARTITEMATCHINGS by adding vertices to the smaller vertex set. Thus, \#P-hardness of \#SUBTREEs follows from Lemmas 2-4, and from the transitivity of polynomial-time Turing reducibility. We will now show that \#SubTreEs is in \#P. Suppose that $T$ is a tree with vertex set $V_{n}=\left\{v_{0}, \ldots, v_{n-1}\right\}$. We will order the vertices in $V_{n}$ so that $v_{i}<v_{j}$ if and only if $i<j$. For every (labelled) subtree $T^{\prime}$ of $T$, let $V\left(T^{\prime}\right)$ denote the vertex set of $T^{\prime}$. We will say that subtree $T^{\prime \prime}$ is larger than subtree $T^{\prime}$ if and only if there is a vertex $v_{i} \in V_{n}$ such that $v_{i} \in V\left(T^{\prime \prime}\right), v_{i} \notin V\left(T^{\prime}\right)$ and

$$
V\left(T^{\prime}\right) \cap\left\{v_{i+1}, \ldots, v_{n}\right\}=V\left(T^{\prime \prime}\right) \cap\left\{v_{i+1}, \ldots, v_{n}\right\} .
$$

Let $T^{\prime \prime}$ be a subtree of $T$. Either $T^{\prime \prime}$ is the smallest subtree of $T$ in its isomorphism class, or there is a vertex $v_{\ell} \in V\left(T^{\prime \prime}\right)$ such that the sub-forest $F_{\ell}$ of $T$ induced by vertex set

$$
\left\{v_{i} \in V_{n} \mid v_{i}<v_{\ell}\right\} \cup\left\{v_{i} \in V\left(T^{\prime \prime}\right) \mid v_{i}>v_{\ell}\right\}
$$

contains a tree isomorphic to $T^{\prime \prime}$. Thus, one can determine whether $T^{\prime \prime}$ is the smallest subtree of $T$ in its isomorphism class by solving subgraph isomorphism with inputs $F_{\ell}$ and $T^{\prime \prime}$ for all $v_{\ell} \in V\left(T^{\prime \prime}\right)$. Since $F_{\ell}$ is a forest and $T^{\prime \prime}$ is a tree, this can be done in polynomial time [3] using the method of Edmonds and Matula. It is now simple to describe the \#P computation: with input $T$, each branch picks a subtree $T^{\prime \prime}$ of $T$ and rejects unless $T^{\prime \prime}$ is the smallest subtree of $T$ in its isomorphism class.

\section{The reductions}

Denote by $\leqslant_{\mathrm{T}}$ the relation 'is polynomial-time Turing reducible to'.

Lemma 2. \#BipartiteMatchings $\leqslant_{\mathrm{T}}$ \#CommonRootedSubtrees.

Proof. Let $G$ be an instance of \#BipartiteMATChings with vertex sets $\left\{u_{0}, \ldots, u_{n-1}\right\}$ and $\left\{v_{0}, \ldots, v_{n-1}\right\}$. From $G$, we construct two rooted trees, $\left(T_{1}, r_{1}\right)$ and $\left(T_{2}, r_{2}\right)$, each based on a fixed skeleton. The skeleton of $T_{1}$ has vertex set

$$
\left\{x_{i, j}: 0 \leqslant i \leqslant n-1 \text { and } 0 \leqslant j \leqslant n^{2}+i+1\right\} \cup\left\{r_{1}\right\},
$$


$T_{1}$

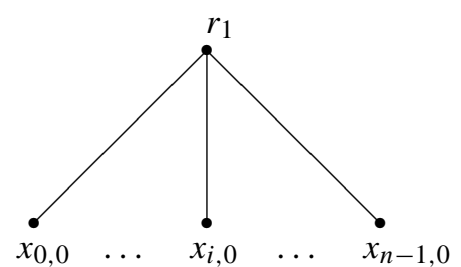

$\begin{array}{lllll}x_{0,0} & \ldots & x_{i, 0} & \ldots & x_{n-1,0}\end{array}$

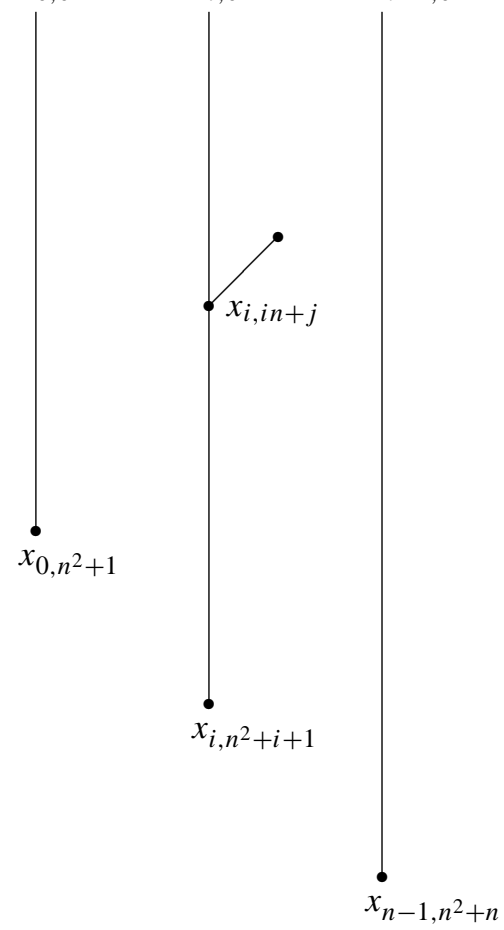

$T_{2}$

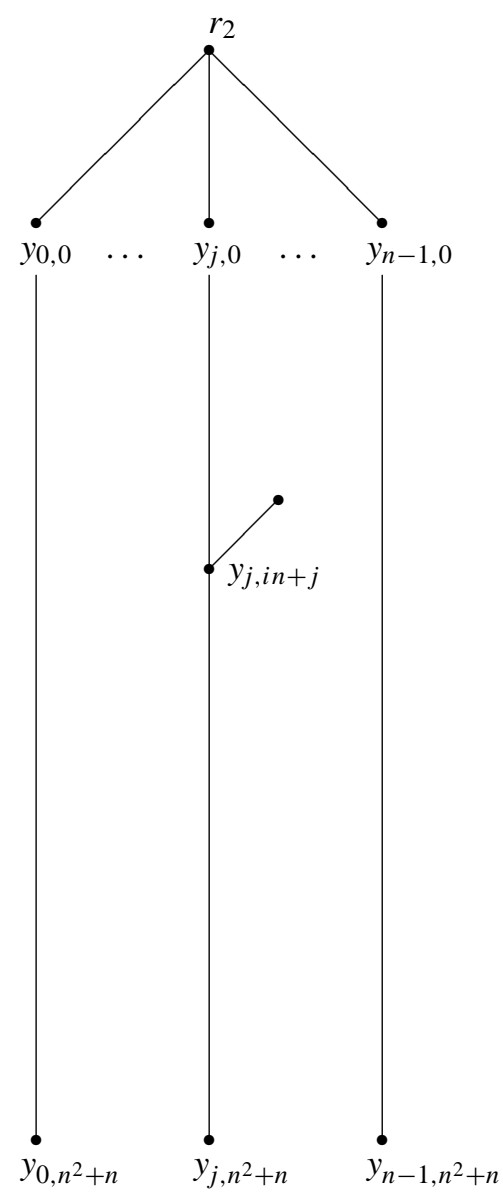

Figure 1: The skeleton of trees $T_{1}$ and $T_{2}$, illustrating the presence of edge $\left(u_{i}, v_{j}\right)$ in $G$.

and edge set

$$
\left\{\left(x_{i, j}, x_{i, j+1}\right): 0 \leqslant i \leqslant n-1 \text { and } 0 \leqslant j \leqslant n^{2}+i\right\} \cup\left\{\left(r_{1}, x_{i, 0}\right): 0 \leqslant i \leqslant n-1\right\} .
$$

Informally, the skeleton of $T_{1}$ consists of $n$ paths of different lengths emanating from the root $r_{1}$, as illustrated in Figure 1. These $n$ paths correspond to the $n$ vertices $\left\{u_{i}\right\}$ of $G$.

The skeleton of $T_{2}$ is similar to the skeleton of $T_{1}$, except that the paths now have equal length. It has vertex set

$$
\left\{y_{i, j}: 0 \leqslant i \leqslant n-1 \text { and } 0 \leqslant j \leqslant n^{2}+n\right\} \cup\left\{r_{2}\right\},
$$

and edge set

$$
\left\{\left(y_{i, j}, y_{i, j+1}\right): 0 \leqslant i \leqslant n-1 \text { and } 0 \leqslant j \leqslant n^{2}+n-1\right\} \cup\left\{\left(r_{2}, y_{i, 0}\right): 0 \leqslant i \leqslant n-1\right\} .
$$

The $n$ paths emanating from $r_{2}$ correspond to the $n$ vertices $\left\{v_{i}\right\}$ of $G$. 
The trees $T_{1}$ and $T_{2}$ are themselves built by adding to the respective skeletons certain edges encoding the graph $G$. Specifically, for each edge $\left(u_{i}, v_{j}\right)$ of $G$, we add an edge from a new vertex to vertex $x_{i, i n+j}$ of $T_{1}$, and add an edge from a new vertex to vertex $y_{j, i n+j}$ of $T_{2}$.

Let $\mathcal{T}^{*}$ denote the set of all finite (unlabelled) rooted trees $(T, r)$ that have leaves at all distances in the range $\left[n^{2}+2, n^{2}+n+1\right]$ from the root $r$. For any rooted tree $(T, r)$, let $\mathcal{T}(T, r)$ denote the set of all (unlabelled) rooted subtrees of $(T, r)$. Thus, the quantity \#RootedSubtrees $(T, r)$ is just the size of $\mathcal{T}(T, r)$. We first observe that there is a bijection between the set of matchings (of all sizes) in $G$ and the set $\mathcal{T}\left(T_{1}, r_{1}\right) \cap \mathcal{T}\left(T_{2}, r_{2}\right) \cap \mathcal{T}^{*}$, and then conclude the proof by showing how to compute the size of $\mathcal{T}\left(T_{1}, r_{1}\right) \cap \mathcal{T}\left(T_{2}, r_{2}\right) \cap \mathcal{T}^{*}$ using an oracle for \#COMmONRoOTEDSUbTREES.

Consider some tree $(T, r) \in \mathcal{T}\left(T_{1}, r_{1}\right) \cap \mathcal{T}\left(T_{2}, r_{2}\right) \cap \mathcal{T}^{*}$. From the definition of $\mathcal{T}^{*}$ we see that $T$ must contain the entire skeleton of $T_{1}$. Let us now see which other edges of $T_{1}$ can be present in $T$. That is, we will now consider the 'pendant edges' which hang off of the skeleton of $T_{1}$. Suppose that for some $i$ and $j$ in $\{0, \ldots, n-1\}$ there is a pendant edge $e$ at distance $i n+j+1$ from the root of $T$. Then the edge $\left(u_{i}, v_{j}\right)$ must be present in $E(G)$. Also, for any $j^{\prime} \in\{0, \ldots, n-1\}$ which is not equal to $j, T$ cannot contain a pendant edge $e^{\prime}$ at distance $i n+j^{\prime}+1$ from the root. (To see this, note that by the construction of $T_{1}$, edge $e^{\prime}$ would be a descendant of $x_{i, 0}$ in $T_{1}$. The presence of $e$ in $T$ ensures that $x_{i, 0}$ and $y_{j, 0}$ are associated with the same vertex of $T$ but $e^{\prime}$ is not a descendant of $y_{j, 0}$ in $T_{2}$.) Similarly, for any $i^{\prime} \in\{0, \ldots, n-1\}$ which is not equal to $i, T$ cannot contain a pendant edge $e^{\prime}$ at distance $i^{\prime} n+j+1$ from the root. Thus, $T$ contains at most $n$ pendant edges, and these correspond to a matching in $E(G)$. So, every rooted tree $(T, r) \in \mathcal{T}\left(T_{1}, r_{1}\right) \cap \mathcal{T}\left(T_{2}, r_{2}\right) \cap \mathcal{T}^{*}$ may be interpreted as a matching in $G$, and vice versa. This is the sought-for bijection between the set of matchings in $G$ and the set $\mathcal{T}\left(T_{1}, r_{1}\right) \cap \mathcal{T}\left(T_{2}, r_{2}\right) \cap \mathcal{T}^{*}$. To conclude, we just need to show how to compute the size of the latter set using an oracle for \#COMMONROOTEDSUBTREES.

Let $L$ be the set of all leaves in $\left(T_{1}, r_{1}\right)$ whose distances from the root $r_{1}$ are in the range $\left[n^{2}+2, n^{2}+n+1\right]$. Let $U$ be the set of all vertices in $\left(T_{2}, r_{2}\right)$ whose distances from $r_{2}$ are in the range $\left[n^{2}+2, n^{2}+n+1\right]$. For each $j \in\{0, \ldots, n\}$, let $T_{1}^{j}$ be the tree formed from $\left(T_{1}, r_{1}\right)$ by adorning every vertex in $L$ with a tuft of $n+j$ edges, and let $T_{2}^{j}$ be the tree formed from $\left(T_{2}, r_{2}\right)$ by adorning every vertex in $U$ with a tuft of $n+j$ edges. By the phrase 'adorning a vertex $v$ with a tuft of $t$ edges' we mean the following: 'create $t$ new vertices and add an edge from each of these new vertices to $v$ '. For $k \in\{0, \ldots, n\}$, let $a_{k}$ be the number of rooted trees in $\mathcal{T}\left(T_{1}^{0}, r_{1}\right) \cap \mathcal{T}\left(T_{2}^{0}, r_{2}\right)$ that have $k$ vertices of degree $n+1$. Clearly,

$$
a_{n}=\left|\mathcal{T}\left(T_{1}, r_{1}\right) \cap \mathcal{T}\left(T_{2}, r_{2}\right) \cap \mathcal{T}^{*}\right| .
$$

So we want to show how to compute $a_{n}$ using an oracle for \#CommonRootedSubtrees.

We claim (and shall presently justify) that

$$
\left|\mathcal{T}\left(T_{1}^{j}, r_{1}\right) \cap \mathcal{T}\left(T_{2}^{j}, r_{2}\right)\right|=\sum_{k=0}^{n} a_{k}(j+1)^{k} .
$$

Thus, we can use an oracle for \#COMmonRootedSubtrees to evaluate the left-hand side of 1 at $j=0, \ldots n$; then we can compute $a_{n}$ by Lagrange interpolation. (See [11] for details of this process, particularly the claim that interpolation is a polynomial-time operation.)

It remains to prove equation (1). We define a projection function

$$
\pi: \mathcal{T}\left(T_{1}^{j}, r_{1}\right) \cap \mathcal{T}\left(T_{2}^{j}, r_{2}\right) \rightarrow \mathcal{T}\left(T_{1}^{0}, r_{1}\right) \cap \mathcal{T}\left(T_{2}^{0}, r_{2}\right)
$$


as follows. For any rooted tree $(T, r)$ in the domain, $\left(T^{\prime}, r\right)=\pi(T, r)$ is the maximum $r$-rooted subtree of $(T, r)$ that has no vertex of degree greater than $n+1$. To see that $T^{\prime}$ is uniquely defined, consider an embedding of $(T, r)$ into $\left(T_{1}^{j}, r_{1}\right)$. The only vertices of degree greater than $n+1$ are those that are mapped to tufts. Thus, $\left(T^{\prime}, r\right)$ is obtained from $(T, r)$ by pruning tufts with more than $n$ pendant edges, down to exactly $n$ pendant edges. Note also that the resulting tree $\left(T^{\prime}, r\right)$ can be embedded in both $\left(T_{1}^{0}, r_{1}\right)$ and $\left(T_{2}^{0}, r_{2}\right)$, so $\pi$ is indeed well defined.

How large is $\pi^{-1}\left(T^{\prime}, r\right)$ ? To every tuft with exactly $n$ pendant edges we may add any number of pendant edges, from 0 to $j$. All the tufts are distinguishable, because they are all at distinct distances from the root $r$. Thus all these possible augmentations lead to distinct trees, and $\pi^{-1}\left(T^{\prime}, r\right)=(j+1)^{k}$, where $k$ is the number of vertices in $\left(T^{\prime}, r\right)$ of degree $n+1$. Thus, each of the $a_{k}$ rooted trees in $\mathcal{T}\left(T_{1}^{0}, r_{1}\right) \cap \mathcal{T}\left(T_{2}^{0}, r_{2}\right)$ with $k$ vertices of degree $n+1$ is mapped by $\pi^{-1}$ to $(j+1)^{k}$ trees in $\mathcal{T}\left(T_{1}^{j}, r_{1}\right) \cap \mathcal{T}\left(T_{2}^{j}, r_{2}\right)$. The lemma follows.

\section{Lemma 3. \#COMmonRootedSubtrees $\leqslant \mathrm{T}$ \#RootedSubtrees.}

Proof. Suppose that $\left(T_{1}, r_{1}\right)$ and $\left(T_{2}, r_{2}\right)$ constitute an instance of \#COMMONRootedSuBTREES. Let $(T, r)$ be the rooted tree formed by taking $T_{1}$ and $T_{2}$ and adding a new root, $r$, and edges $\left(r, r_{1}\right)$ and $\left(r, r_{2}\right)$. For notational convenience, introduce the following quantities:

$$
\begin{aligned}
N_{1} & =\text { \#RootedSubtrees }\left(T_{1}, r_{1}\right) \\
N_{2} & =\text { \#RootedSubtrees }\left(T_{2}, r_{2}\right), \\
N & =\text { \#RootedSubtrees }(T, r), \text { and } \\
C & =\text { \#CommonRootedSubtrees }\left(\left(T_{1}, r_{1}\right),\left(T_{2}, r_{2}\right)\right)
\end{aligned}
$$

We start by observing that

$$
N=1+N_{1}+N_{2}-C+N_{1} N_{2}-\left(\begin{array}{l}
C \\
2
\end{array}\right) .
$$

To see this, note that $(T, r)$ has

- one distinct subtree in which the degree of $r$ is 0 , and

- $N_{1}+N_{2}-C$ distinct subtrees in which the degree of $r$ is 1 , and

- $N_{1} N_{2}-\left(\begin{array}{l}C \\ 2\end{array}\right)$ distinct subtrees in which the degree of $r$ is 2 .

Thus, $C(C+1)=2 Z$, where $Z$ denotes

$$
1+N_{1}+N_{2}+N_{1} N_{2}-N .
$$

To compute $C$, first calculate $Z$ using an oracle for \#ROOTEDSubTREES. Then, observe that

$$
C^{2}<2 Z<(C+1)^{2}
$$

so $C$ is the integer square root of $2 Z$, which can be computed in $\Theta(\log Z)$ time. Note that $\log Z$ is polynomially bounded in the size of the input, since, for example, $N_{1} \leqslant 2^{n_{1}}$, where $n_{1}$ is the number of vertices in $T_{1}$.

\section{Lemma 4. \#RootedSubtrees $\leqslant \mathrm{T}$ \#Subtrees.}

Proof. For any $i$, an ' $i$-tuft' is a tree consisting of one (centre) vertex with degree $i$ and $i$ (outer) vertices, each of which has degree 1. 
Suppose that $(T, r)$ is an instance of \#RootedSubTrEes. Let $\Delta$ denote the maximum degree of a vertex in $T$. Let $T^{\prime}$ be the tree formed from $T$ by taking a new $(\Delta+3)$-tuft, and identifying one of the outer vertices with $r$. Let $T^{\prime \prime}$ be the tree formed from $T$ by taking a new $(\Delta+2)$-tuft, and identifying one of the outer vertices with $r$. Let $N^{\prime}$ denote \#SUBTREES $\left(T^{\prime}\right)$, and let $N^{\prime \prime}$ denote \#Subtrees $\left(T^{\prime \prime}\right)$. Then \#RootedSubtrees $(T, r)$ is equal to $N^{\prime}-N^{\prime \prime}$, so it can be computed using an oracle for \#SUBTREES.

\section{Some consequences}

Following Valiant [11], we say that a function $f: \Sigma^{*} \rightarrow \mathbb{N}$ is in FP if it can be computed by a deterministic polynomial-time Turing machine. We say that it is in $\mathrm{FP}^{g}$ for a problem $g$ if it can be computed by a deterministic polynomial-time Turing machine which is equipped with an oracle for $g$. Finally, we say that it is in $\mathrm{FP}^{A}$ for a complexity class $A$ if there is some $g \in A$ such that $f \in \mathrm{FP}^{g}$.

Let \#CONNECTEDSUBGRAPHS be the problem of counting unlabelled connected subgraphs of a graph. Formally, let it be defined as follows.

\section{Name. \#CONNECTEDSUBGRAPHS}

Instance. A graph $G$.

Output. The number of distinct (up to isomorphism) connected subgraphs of $G$.

\section{Corollary 5. \#CONNECTEDSUBGRAPHS is complete for $\mathrm{FP}^{\# \mathrm{P}}$.}

Proof. \#CONnECTEdSubgraphs is FP \#P -hard by Theorem 1. We will show that \#ConNECTEDSUBGRAPHS is in the class FP ${ }^{\text {span-P }}$, which will be defined shortly. The result will then follow by Toda's theorem [9].

We start by defining the relevant complexity classes. A function $f: \Sigma^{*} \rightarrow \mathbb{N}$ is in the class span-P [7] if there is a polynomial-time nondeterministic Turing machine $M$ (with an output device) such that the number of different accepting outputs of $M$ on input $x$ is $f(x)$, for all $x \in \Sigma^{*}$.

A function $f: \Sigma^{*} \rightarrow \mathbb{N}$ is in \#NP if there is a polynomial-time nondeterministic Turing machine $M$ and an oracle $A \in \mathrm{NP}$ such that the number of accepting computations of $M^{A}$ on input $x$ is $f(x)$, for all $x \in \Sigma^{*}$.

The classes \#P, span-P, and \#NP are related [7] by

$$
\# \mathrm{P} \subseteq \text { span-P } \subseteq \# \mathrm{NP} .
$$

Thus,

$$
\mathrm{FP}^{\# \mathrm{P}} \subseteq \mathrm{FP}^{\mathrm{span}-\mathrm{P}} \subseteq \mathrm{FP}^{\# \mathrm{NP}}
$$

But FP $\#$ NP $\subseteq \mathrm{FP}^{\# \mathrm{PH}}$, where \#PH is the class of functions that count the number of accepting computations of polynomial-time nondeterministic Turing machines with oracles from PH. Furthermore, Toda and Watanabe [10] show \#PH $\subseteq \mathrm{FP}^{\# \mathrm{P}}$. Thus,

$$
\mathrm{FP}^{\# \mathrm{P}}=\mathrm{FP}^{\mathrm{span}-\mathrm{P}} \text {. }
$$

(See also Section 1.8 of Welsh's book [12].)

We now complete the proof by showing that \#CONNECTEDSUBGRAPHS is in FP ${ }^{\text {span-P }}$. Let $N(G, k)$ denote $k$ ! times the number of distinct (up to isomorphism) connected size- $k$ 
subgraphs of $G$. Since

$$
\text { \#ConnectedSubgraphs }(G)=\sum_{k=1}^{n} \frac{1}{k !} N(G, k),
$$

where $n$ is the number of vertices of $G$, it suffices to show that computing $N(G, k)$ is in span-P. Each branch of the computation tree for $N(G, k)$ chooses

- a size- $k$ connected subgraph $H$ of $G$,

- a bijection $\sigma$ from the vertices of $H$ to the set $\left\{v_{1}, \ldots, v_{k}\right\}$, and

- a permutation $\pi$ of $v_{1}, \ldots, v_{k}$.

Let $H^{\prime}$ be the graph formed from $H$ by relabelling each vertex $v$ of $H$ with the label $\sigma(v)$. If $\pi$ is an automorphism of $H^{\prime}$ then $\left(H^{\prime}, \pi\right)$ is output. Otherwise, the branch rejects. The result now follows from Burnside's lemma, which implies that for any given isomorphism class of $k$-vertex graphs, the number of graphs in the isomorphism class times the number of automorphisms of any member of the class is equal to $k$ !. (For example, see [5].)

Let \#GRAPHSUBTREES be the problem of counting unlabelled subtrees of a graph. Formally, let it be defined as follows.

Name. \#GraphSubTreEs

Instance. A graph $G$.

Output. The number of distinct (up to isomorphism) subtrees of $G$.

Corollary 6. \#GRAPHSUBTREES is complete for $\mathrm{FP}^{\# P}$.

Proof. This is the same as the proof of Corollary 5, except that the span-P computation rejects any subgraph $H$ which is not a tree. A more direct proof could be obtained by using a polynomial-time canonical labelling algorithm for trees such as the one by Hopcroft and Tarjan [6].

\section{References}

1. G. Blache, M. Karpinski and J. Wirtgen, 'On approximation intractability of the bandwidth problem', Electronic Colloquium on Computational Complexity, Report TR98-014, 1998. http://www.eccc.uni-trier.de/eccc-local/Lists/TR-1998.html 117

2. K. J. Edwards and C. J. H. McDiarmid, 'The complexity of harmonious colouring for trees', Discrete Appl. Math. 57 (1995) 133-144. 117

3. M. R. GAREY and D. S. Johnson, Computers and intractability: a guide to the theory of NP-completeness (Freeman, San Francisco, CA, 1979). 117, 118

4. M. R. Garey, R. L. Graham, D. S. Johnson and D. E. Knuth, 'Complexity results for bandwidth minimization', SIAM J. Appl. Math. 34 (1978) 477-495. 117

5. F. Harary and E. M. Palmer, Graphical enumeration (Academic Press, 1973). 123

6. J. E. Hopcroft and R. E. TARJAN, 'Efficient planarity testing', J. ACM 21 (1974) 549-568. 123

7. J. KöBlER, U. SchöNING and J. TORAN, 'On counting and approximation', Acta Inform. 26 (1989) 363-379. 122, 122

8. C. H. Papadimitriou, Computational complexity (Addison-Wesley, 1994). 117 
9. S. Toda, 'PP is as hard as the polynomial-time hierarchy', SIAM J. Comput. 20 (1991) 865-877. 122

10. S. Toda and O. Watanabe, 'Polynomial-time 1-Turing reductions from \#PH to \#P', Theoret. Comput. Sci. 100 (1992) 205-221. 122

11. L. G. Valiant, 'The complexity of enumeration and reliability problems', SIAM J. Comput. 8 (1979) 410-421. 117, 118, 120, 122

12. D. J. A. Welsh, Complexity: knots, colourings and counting (Cambridge University Press, 1993). 122

Leslie Ann Goldberg leslie@dcs.warwick.ac.uk

Department of Computer Science, University of Warwick, Coventry, CV4 7AL

http://www.dcs.warwick.ac.uk/ leslie/

Mark Jerrum mrj@dcs.ed.ac.uk

Department of Computer Science, University of Edinburgh, The King's Buildings, Edinburgh EH9 3JZ

http://www.dcs.ed.ac.uk/ mrj/ 